\title{
Cost-effectiveness of primary debulking surgery when compared to neoadjuvant chemotherapy in the management of stage IIIC and IV epithelial
} ovarian cancer

\author{
This article was published in the following Dove Press journal: \\ ClinicoEconomics and Outcomes Research \\ 2 August 2016 \\ Number of times this article has been viewed
}

\author{
Gareth K Forde' \\ Jenny Chang ${ }^{2}$ \\ Argyrios Ziogas ${ }^{2}$ \\ 'Division of Gynecologic Oncology, \\ Department of Obstetrics and \\ Gynecology, Irvine Medical Center, \\ University of California, Orange, CA, \\ USA; ${ }^{2}$ Department of Epidemiology, \\ University of California, Irvine, \\ CA, USA
}

Objectives: To examine the cost-effectiveness of primary debulking surgery (PDS) when compared to neoadjuvant chemotherapy (NACT) in the management of epithelial ovarian cancer (EOC) using Surveillance, Epidemiology, and End Results data linked to Medicare claims (SEER-Medicare).

Methods: Using a Markov model, the cost-effectiveness of PDS was compared to that of NACT. We modeled cost and survival inputs using data from women in the SEER-Medicare database with ovarian cancer treated by either PDS or NACT between 1992 and 2009. Direct and indirect costs were discounted by an annual rate of $3 \%$. Utility weights were obtained from published data. The incremental cost-effectiveness ratio (ICER) of PDS compared to NACT was calculated.

Results: In our model, women with stage IIIC EOC had a higher mean adjusted treatment cost for PDS when compared to NACT $(\$ 31,945$ vs $\$ 30,016)$ but yielded greater qualityadjusted life-years (QALYs) (1.79 vs 1.69). The ICER was \$19,359/QALY gained. Women with stage IV EOC had a higher mean adjusted treatment cost following PDS when compared to NACT $(\$ 31,869$ vs $\$ 27,338)$ but yielded greater QALYs (1.69 vs 1.66$)$. The ICER was $\$ 130,083 /$ QALY gained. A sensitivity analysis showed that for both PDS and NACT the ICER was sensitive to incremental changes in the utility weight.

Conclusion: PDS is significantly more cost-effective for women with stage IIIC when compared to NACT. In women with stage IV EOC, PDS is also more cost-effective though the QALYs gained are much more costly and exceed a $\$ 50,000$ willingness to pay.

Keywords: Markov model, gynecologic cancer, chemotherapy, up front surgery

\section{Introduction}

In 2016, it is estimated that more than 22,000 women will be diagnosed with ovarian cancer. Of these, over 14,000 are expected to die from it. In women aged 40-59 and 60-79 years, ovarian cancer is the fourth and fifth leading cause of cancer-related death, respectively, and is the leading cause of death from a gynecologic malignancy for all women in the USA. Over the past 40 years, the 5-year relative survival rate has only slightly increased. ${ }^{1}$

Ovarian cancer is associated with poor survival because it is usually diagnosed at an advanced stage. Because most women present with disease that extends beyond the pelvis, the 5-year survival rates remain less than 50\%. Ovarian cancer survival is associated with a number of addressable factors, including: 1) timing to initiation of
Correspondence: Gareth K Forde Division of Gynecologic Oncology, Department of Obstetrics and Gynecology, Irvine Medical Center, University of California, I0I The City

Drive, Orange, CA 92868, USA

Tel + I 7l4456 8020

Email gforde@uci.edu 
chemotherapy following cytoreductive surgery; ${ }^{2} 2$ ) administration of doublet chemotherapy with a platinum-based agent and a taxane; ${ }^{3}$ and 3 ) reduction of tumor burden by surgical debulking to no grossly visible residual disease. ${ }^{4}$

According to National Comprehensive Cancer Network (NCCN) guidelines, the primary treatment for ovarian cancer is primary debulking surgery (PDS), followed by platinumbased chemotherapy. However, in certain circumstances, women are treated with chemotherapy first followed by surgery. A clinician's decision to administer chemotherapy, also referred to as neoadjuvant chemotherapy (NACT), prior to cytoreductive surgery has also been evaluated as a factor associated with survival. ${ }^{3}$

Several retrospective analyses have supported a survival advantage associated with PDS..$^{5-7}$ Conversely, two prospective trials, the Chemotherapy or Upfront Surgery (CHORUS) trial and the European Organisation for Research and Treatment of Cancer (EORTC) trial, showed an equivalent survival but increased morbidity with PDS when compared to NACT. ${ }^{7-9}$ In a more recent retrospective analysis of Surveillance, Epidemiology, and End Results data linked to Medicare claims (SEER-Medicare) data, Wright et al found that both treatments conveyed an equivalent survival advantage with a decrease in morbidity associated with NACT. ${ }^{10}$

The costs associated with caring for women with advanced ovarian cancer include those incurred during the initial treatment, treatment of recurrences, and end-of-life care. To date, prior studies have not evaluated the cost-effectiveness of PDS compared to NACT. A cost-effectiveness analysis may provide additional insights into the overall benefit of each treatment strategy by comparing costs relative to survival. In a cost-effectiveness analysis, factors such as quality of life, cost per quality-adjusted life-year (QALY) gained, and survival can be integrated to provide a more complete interpretation. The purpose of this investigation is to evaluate the cost-effectiveness of PDS when compared to NACT in women with advanced epithelial ovarian cancer (EOC) using survival and cost assumptions from SEER-Medicare data.

\section{Methods}

The study design was a retrospective population-based study using the SEER-Medicare database. University of California, Irvine Institutional Review Board approval was obtained (HS\#2012-9076). Patient consent was not deemed necessary by the IRB. The SEER Database of the National Cancer Institute (NCI) contains approximately $97 \%$ of all incident cancer cases from tumor registries that covered $14 \%$ of the US population in 1995 to $28 \%$ currently. ${ }^{11-13}$
The SEER Program registries collect data on patient demographics, primary tumor site, tumor morphology and stage, first course of treatment, and follow-up for vital status. Among patients older than 65 years in SEER data, 93\% were identified in the Medicare enrolment file and their records were successfully matched to SEER cases in the linkage process performed by the NCI and the Centers for Medicare and Medicaid Services. ${ }^{11,12}$ The Medicare claims database includes all inpatient hospitalizations, outpatient visits, physician/supplier data, durable medical equipment, and hospice and home health care. All claims are longitudinal from the time of a person's Medicare eligibility until death. Our analysis data include SEER cases from 1992 to 2009 and their Medicare claims from 1991 to 2010 .

\section{Study population}

A total of 38,792 patients diagnosed between January 1, 1992 and December 31, 2009 with invasive ovarian cancer (SEER primary site code C569) as their only tumor or first primary tumor with the second tumor at least 2 years after the first ovarian cancer were identified in SEER data. We sequentially removed 287 cases with missing tumor histology information, 117 with germ cell or sex cord tumor, 820 with autopsy or death certificate only, 10,468 with age at diagnosis less than 66 years, 3,337 with missing tumor stage information, 8,731 with early stage (stage IIIB and below), 36 with missing diagnosis month, and 4,696 without continuous enrolment of Medicare Part A and Part B or who were ever enrolled in a health maintenance organization from the 12 th month prior to diagnosis. Using the International Classification of Disease, Ninth Revision, Clinical Modification (ICD-9-CM) procedure and diagnosis codes in claims data (Table S1), 4,714 patients who had received both ovarian cancer surgery and chemotherapy treatments were identified. In order to account for survival bias and capture true treatment costs, patients observed for less than 5 months after cancer diagnosis were excluded, which resulted in excluding 196 patients, and the final study population consisted of 4,518 patients.

The surgery date was estimated by the admission date of inpatient stay for surgery. The starting date of chemotherapy was estimated by the date of first chemotherapy claim after cancer diagnosis. Patients who had an earlier surgery date or the same surgery and chemotherapy dates were placed in the PDS group and patients with earlier chemotherapy start dates were placed in the NACT group. Patients who started chemotherapy during the hospital stay for the surgery were identified as PDS. 


\section{Survival}

Patients' survival time was defined as the time between pathologic diagnosis and death or last follow-up. Survival estimates were based on final analysis data of 4,518 patients who were identified as having either PDS or NACT (Table 1). The Kaplan-Meier method and log-rank tests were used to estimate survival probability. Year 1-5 survival rates were calculated, and the Kaplan-Meier survival curves are given in Figure 1. The time horizon of 60 months was selected to correlate with 5-year survival data.

\section{Costs}

Costs in US dollars for patients treated by PDS or NACT with stages IIIC and IV ovarian cancer are shown in Tables 2 and 3. The costs were discounted by 3\%. A 7-month time horizon was selected to represent costs associated with the initial treatment by PDS or NACT and to exclude downstream costs related to second-line treatment, palliation, or end-of-life care. The total cost of treatment was calculated as a summation of the costs incurred during the 7 months following initial diagnosis: 1) hospital inpatient costs; 2) physician costs; 3) outpatient costs; 4) hospice costs; 5) home health costs; and 6) durable medical equipment costs. Costs associated with the treatment of recurrence and end-of-life care were assumed to be equivalent for PDS and NACT. Costs were inflation adjusted and presented in 2010 US dollars using the consumer price index for medical care services from the United States Bureau of Labor Statistics. ${ }^{14}$

\section{Quality of life}

Quality of life estimates were adjusted using standard utility weights. Estimates assume that 1 year of perfect health is assigned a utility weight of 1 and that 1 year of imperfect health is assigned a utility weight of less than 1 . In a theoretical patient with perfect health over a 5-year survival period, their survival QALY would equal 5. Conversely, if the same patient had a survival of 5 years but these were spent in a state of imperfect health with a utility weight of 0.5 , then they would have a QALY equal to 2.5. In this analysis, utility weights were taken from published studies. ${ }^{15-17}$ The utility weight for chemotherapy was 0.77 for the first $1-5$ months and 0.84 for months $6-12$. An average $(0.81)$ was taken to represent the 12-month period following diagnosis. A utility weight of 0.94 was used to represent living with ovarian

Table I Model estimates: clinical assumptions

\begin{tabular}{|c|c|c|}
\hline Clinical parameter & Model estimate & Reference \\
\hline \multicolumn{3}{|l|}{ Survival assumptions } \\
\hline \multicolumn{3}{|l|}{ Stage IIIC survival ovarian cancer } \\
\hline I-year survival treated with PDS & $90.7 \%$ & National Cancer Institute ${ }^{26}$ \\
\hline 2-year survival treated with PDS & $70.5 \%$ & National Cancer Institute ${ }^{26}$ \\
\hline 3-year survival treated with PDS & $54.3 \%$ & National Cancer Institute ${ }^{26}$ \\
\hline 4-year survival treated with PDS & $41.2 \%$ & National Cancer Institute ${ }^{26}$ \\
\hline 5-year survival treated with PDS & $33.4 \%$ & National Cancer Institute ${ }^{26}$ \\
\hline I-year survival treated with NACT & $89.7 \%$ & National Cancer Institute ${ }^{26}$ \\
\hline 2-year survival treated with NACT & $65.6 \%$ & National Cancer Institute ${ }^{26}$ \\
\hline 3-year survival treated with NACT & $41.8 \%$ & National Cancer Institute ${ }^{26}$ \\
\hline 4-year survival treated with NACT & $32.3 \%$ & National Cancer Institute ${ }^{26}$ \\
\hline 5-year survival treated with NACT & $24.1 \%$ & National Cancer Institute ${ }^{26}$ \\
\hline \multicolumn{3}{|l|}{ Stage IV survival ovarian cancer } \\
\hline I-year survival treated with PDS & $87.2 \%$ & National Cancer Institute ${ }^{26}$ \\
\hline 2-year survival treated with PDS & $62.1 \%$ & National Cancer Institute 26 \\
\hline 3-year survival treated with PDS & $45.2 \%$ & National Cancer Institute ${ }^{26}$ \\
\hline 4-year survival treated with PDS & $33.2 \%$ & National Cancer Institute ${ }^{26}$ \\
\hline 5-year survival treated with PDS & $24.7 \%$ & National Cancer Institute 26 \\
\hline I-year survival treated with NACT & $88.8 \%$ & National Cancer Institute ${ }^{26}$ \\
\hline 2-year survival treated with NACT & $60.2 \%$ & National Cancer Institute ${ }^{26}$ \\
\hline 3-year survival treated with NACT & $40.6 \%$ & National Cancer Institute ${ }^{26}$ \\
\hline 4-year survival treated with NACT & $25.4 \%$ & National Cancer Institute ${ }^{26}$ \\
\hline 5-year survival treated with NACT & $17.7 \%$ & National Cancer Institute ${ }^{26}$ \\
\hline \multicolumn{3}{|l|}{ Utility weights } \\
\hline Months I-5 following chemotherapy & 0.77 & Chi et $\mathrm{al}^{5}$ \\
\hline Months 6-12 following chemotherapy & 0.88 & Chi et $\mathrm{al}^{5}$ \\
\hline Year 2 living with ovarian cancer & 0.94 & Chi et $\mathrm{al}^{5}$ \\
\hline Years 3-5 living with ovarian cancer & 0.5 & Chi et $\mathrm{al}^{5}$ \\
\hline
\end{tabular}

Abbreviations: NACT, neoadjuvant chemotherapy; PDS, primary debulking surgery. 


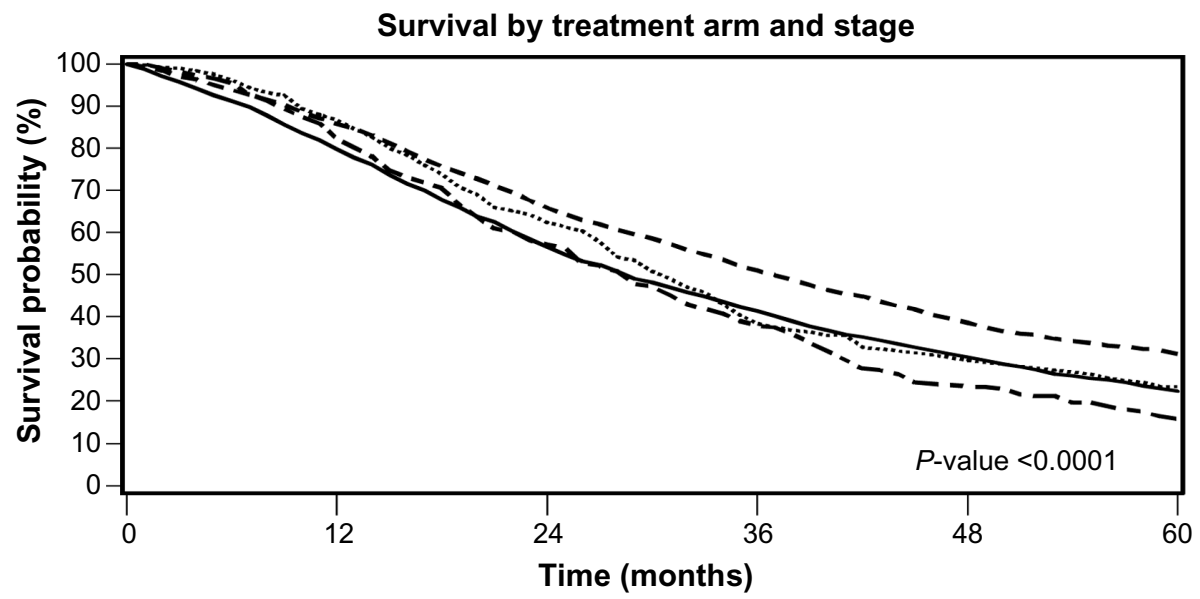

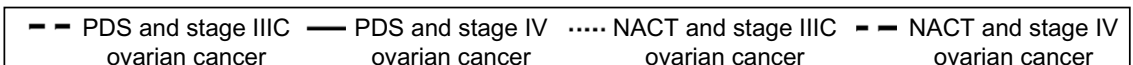

Figure I Kaplan-Meier survival curves for each treatment strategy.

Abbreviations: NACT, neoadjuvant chemotherapy; PDS, primary debulking surgery.

cancer during the second year following diagnosis. A utility weight of 0.5 was used to represent the impact of cancer progression during years 3 through 5 .

\section{Analysis}

The economic analysis was performed using TreeAge Pro Software $^{\circledR}$ (TreeAge Software Inc., Williamstown, MA, USA). ${ }^{18}$ A decision tree with a Markov state transition model was constructed to compare the cost-effectiveness of NACT and PDS in stage IIIC and IV EOC (Figures 1 and 2), respectively. Transition state probabilities and cost estimates were obtained using the SEER-Medicare database. The time horizon was 5 years. Effectiveness was measured in QALYs. The model classified subjects into two health states: alive with ovarian cancer and dead. Because there is a time value of money, direct and indirect costs associated with each intervention were discounted by an annual rate of $3 \%$ to determine their present value. The utility weights were taken from published data. ${ }^{19}$

The model assumptions include: 1) the major treatmentrelated costs of PDS and NACT occur during the first 7 months of treatment; 2) quality-of-life utility weights change with disease progression; and 3) there are no significant differences in the quality of life between PDS and NACT. A sensitivity analysis was performed to evaluate how numerical uncertainty can be apportioned to NACT and PDS.

\section{Results}

\section{Base case}

Survival and cost estimates were obtained from SEERMedicare data for patients treated with PDS and NACT. Of the 4,518 women deemed eligible for this analysis, $82.4 \%$ underwent PDS and $17.6 \%$ received NACT. Of women with stage IIIC and IV EOC, 85.4\% and 78.5\% received PDS, respectively. Survival data for years 1 through 5 were used at Markov transition state nodes. NACT was associated with a decreased 5-year overall survival for stage IIIC and IV EOC when compared to PDS. The hazard ratios were 1.36 and 1.28 for stage IIIC and IV EOC, respectively. The 7-month treatment costs for stage IIIC patients treated with NACT or PDS were $\$ 59,769$ and $\$ 59,805$, respectively (Table 2). For women with stage IV EOC, the treatment costs were $\$ 60,442$ and $\$ 63,067$ for NACT and PDS, respectively.

\section{Stage IIIC EOC}

The cost of NACT when compared to PDS for women with stage IIIC EOC, using the model, is shown in Table 4. The cost of NACT was $\$ 30,016$ compared to $\$ 31,945$ for PDS. The incremental cost was $\$ 1,929$ (Table 4). The effectiveness for NACT was lower than that of PDS (1.69 and 1.79, respectively). The incremental cost-effectiveness ratio (ICER) was $\$ 19,359$. A sensitivity analysis revealed the ICER was sensitive to the utility weight associated with NACT (Figure 2).

\section{Stage IV EOC}

The results of the Markov model for women with stage IV EOC are given in Table 4. The analysis determined that the cost of NACT was $\$ 27,338$ compared to $\$ 31,869$ for PDS in women with stage IV EOC. The incremental cost was $\$ 4,531.50$. The effectiveness of NACT was lower than that of PDS (1.66 and 1.69, respectively). The ICER was \$130,083. 


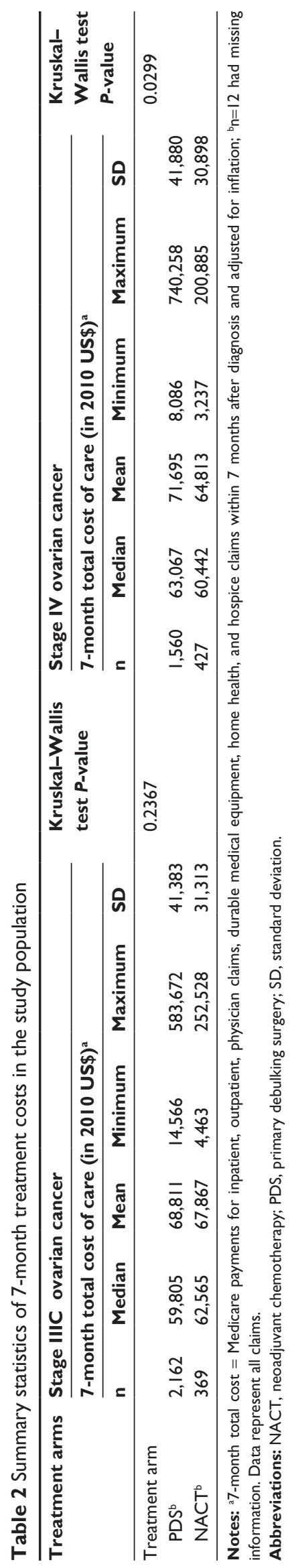

Table 3 Model estimates: costs (2010 US\$)

\begin{tabular}{|c|c|c|}
\hline Clinical parameter & Mean & SD \\
\hline \multicolumn{3}{|l|}{ Stage IIIC ovarian cancer with PDS } \\
\hline 7-month cost - hospital inpatient & $\$ 40,511$ & $\$ 37,365$ \\
\hline 7-month cost - physician claims & $\$ 20,473$ & $\$ 14,528$ \\
\hline 7-month cost - outpatient & $\$ 5,833$ & $\$ 9,483$ \\
\hline 7-month cost - hospice claims & $\$ 74$ & $\$ 985$ \\
\hline 7-month cost - home health & $\$ 1,492$ & $\$ 2,889$ \\
\hline 7-month cost - durable medical equipment & $\$ 427$ & $\$ 2,597$ \\
\hline 7-month total cost & $\$ 68,811$ & $\$ 41,383$ \\
\hline \multicolumn{3}{|l|}{ Stage IIIC ovarian cancer with NACT } \\
\hline 7-month cost - hospital inpatient & $\$ 36,911$ & $\$ 26,106$ \\
\hline 7-month cost - physician claims & $\$ 21,876$ & $\$ 14,176$ \\
\hline 7-month cost - outpatient & $\$ 6,994$ & $\$ 10,689$ \\
\hline 7-month cost - hospice claims & $\$ 21$ & $\$ 255$ \\
\hline 7-month cost - home health & $\$ 1,565$ & $\$ 2,649$ \\
\hline 7 month cost - durable medical equipment & $\$ 499$ & $\$ 2,310$ \\
\hline 7-month total cost & $\$ 67,867$ & $\$ 31,313$ \\
\hline \multicolumn{3}{|l|}{ Stage IV ovarian cancer with PDS } \\
\hline 7-month cost - hospital inpatient & $\$ 44,621$ & $\$ 38,531$ \\
\hline 7-month cost - physician claims & $\$ 20,909$ & $\$ 14,428$ \\
\hline 7-month cost - outpatient & $\$ 4,212$ & $\$ 8,263$ \\
\hline 7-month cost - hospice claims & $\$ 124$ & $\$ 1,299$ \\
\hline 7-month cost - home health & $\$ 1,293$ & $\$ 2,709$ \\
\hline 7-month cost - durable medical equipment & $\$ 536$ & $\$ 3,099$ \\
\hline 7-month total cost & $\$ 71,695$ & $\$ 41,880$ \\
\hline \multicolumn{3}{|l|}{ Stage IV ovarian cancer with NACT } \\
\hline 7-month cost - hospital inpatient & $\$ 35,336$ & $\$ 25,159$ \\
\hline 7-month cost - physician claims & $\$ 20,712$ & $\$ 14,77 \mid$ \\
\hline 7-month cost - outpatient & $\$ 6,919$ & $\$ 9,966$ \\
\hline 7-month cost - hospice claims & $\$ 90$ & $\$ 1,315$ \\
\hline 7 month cost - home health & $\$ 1,201$ & $\$ 2,388$ \\
\hline 7-month cost - durable medical equipment & $\$ 554$ & $\$ 2,525$ \\
\hline 7-month total cost & $\$ 64,813$ & $\$ 30,898$ \\
\hline
\end{tabular}

Abbreviations: NACT, neoadjuvant chemotherapy; PDS, primary debulking surgery; SD, standard deviation.

A sensitivity analysis revealed the ICER was sensitive to the utility weight associated with PDS (Figure 3).

\section{Discussion}

Many studies have reviewed the cost-effectiveness of interventions related to ovarian cancer. ${ }^{15-17,20-24}$ In a recent review article, several critical topics were discussed.$^{24}$ Of these, much of the emphasis was directed toward driving treatment decisions after the initial decision to treat with NACT or perform PDS. What is unfortunate is that, to date, no studies have looked at the cost-effectiveness of the very first decision that is made for patients with ovarian cancer, which is whether to treat with NACT or perform PDS. Moreover, this decision is possibly linked to the overall cost associated with the subsequent downstream decisions and outcomes.

The treatment of advanced ovarian cancer by PDS or NACT has been the subject of debate. Based on the SEERMedicare data used for the assumptions of this analysis, PDS 


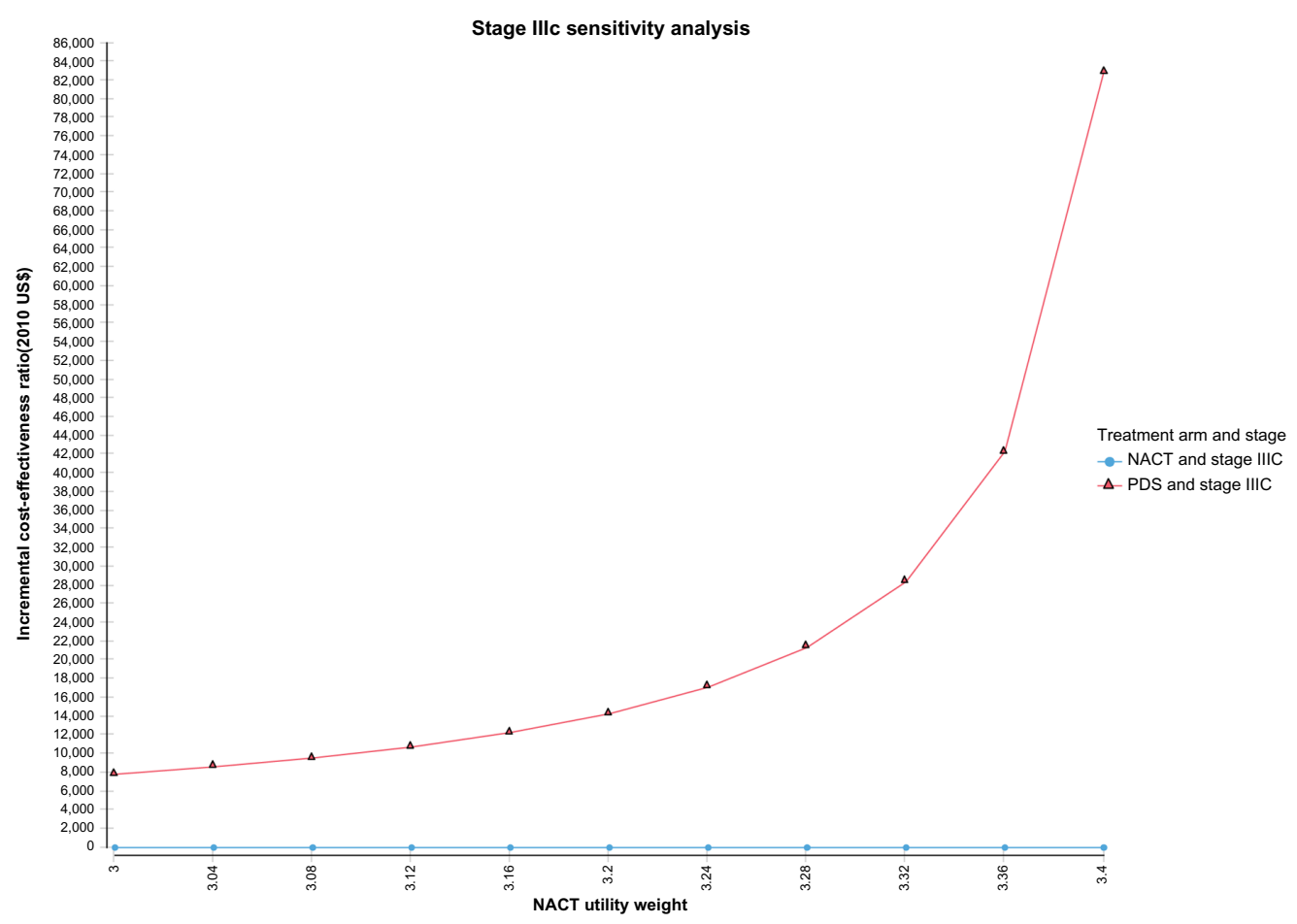

Figure 2 Univariate sensitivity analysis for the impact of the utility weight of NACT on the incremental cost-effectiveness ratio for women with stage IIIC ovarian cancer. Abbreviations: NACT, neoadjuvant chemotherapy; PDS, primary debulking surgery.

offers an increase in 5-year overall survival compared to NACT for both stage IIIC and IV ovarian cancer. In the past, survival comparisons between PDS and NACT have been criticized for a number of reasons, including: 1) physicians selecting sicker patients to NACT over PDS; 2) inability to accurately compare physician surgical proficiency in prior studies; 3) less than average optimal debulking rates; and 4) changes in treatment paradigms over time.

There are several pivotal trials that have looked at survival and morbidity differences between PDS and NACT. In particular, the EORTC study was a prospective trial that randomized women with stage IIIC and IV ovarian cancer to either PDS or NACT. ${ }^{8}$ The analysis concluded that there was no statistically significant difference in mean or overall survival, but that PDS was associated with a higher complication rate. A second analysis, the CHORUS trial, randomized women with stage III and IV ovarian cancer to PDS or NACT. ${ }^{9}$ The results also showed no statistically significant difference in mean and overall survival between PDS and NACT. In addition, women undergoing NACT had a shorter hospital stay. For a number of reasons, both trials have sparked significant debate. First, the optimal debulking rates for the PDS arms are considerably low compared to what are considered standard optimal debulking rates. Also, there are concerns that patients who undergo NACT may be misclassified based on inaccurate pathologic interpretation of the extent of disease because of histologic changes imparted by chemotherapy. Finally, from these studies, it is very difficult to determine the level of surgeon proficiency. It is worth mentioning that a recent retrospective

Table 4 Cost-effectiveness comparison of PDS treatment strategy versus NACT treatment strategy

\begin{tabular}{|c|c|c|c|c|c|}
\hline $\begin{array}{l}\text { Treatment strategy } \\
\text { and cancer stage }\end{array}$ & Cost & Incremental cost & Effectiveness & $\begin{array}{l}\text { Incremental } \\
\text { effectiveness }\end{array}$ & Incremental C/E \\
\hline \multicolumn{6}{|l|}{ Stage IIIC ovarian cancer } \\
\hline NACT & $\$ 30,016$ & & I.69 QALYs & & \\
\hline PDS & $\$ 31,945$ & $\$ 1,929$ & I.79 QALYs & 0.1 QALYs & $\$ 19,359$ \\
\hline \multicolumn{6}{|l|}{ Stage IV ovarian cancer } \\
\hline NACT & $\$ 27,338$ & & I.66 QALYs & & \\
\hline PDS & $\$ 31,869$ & $\$ 4,531$ & I.69 QALYs & 0.03 QALYs & $\$ 130,083$ \\
\hline
\end{tabular}

Note: Currency is 2010 US\$.

Abbreviations: C/E, cost-effectiveness ratio; NACT, neoadjuvant chemotherapy; PDS, primary debulking surgery; QALYs, quality-adjusted life-years. 


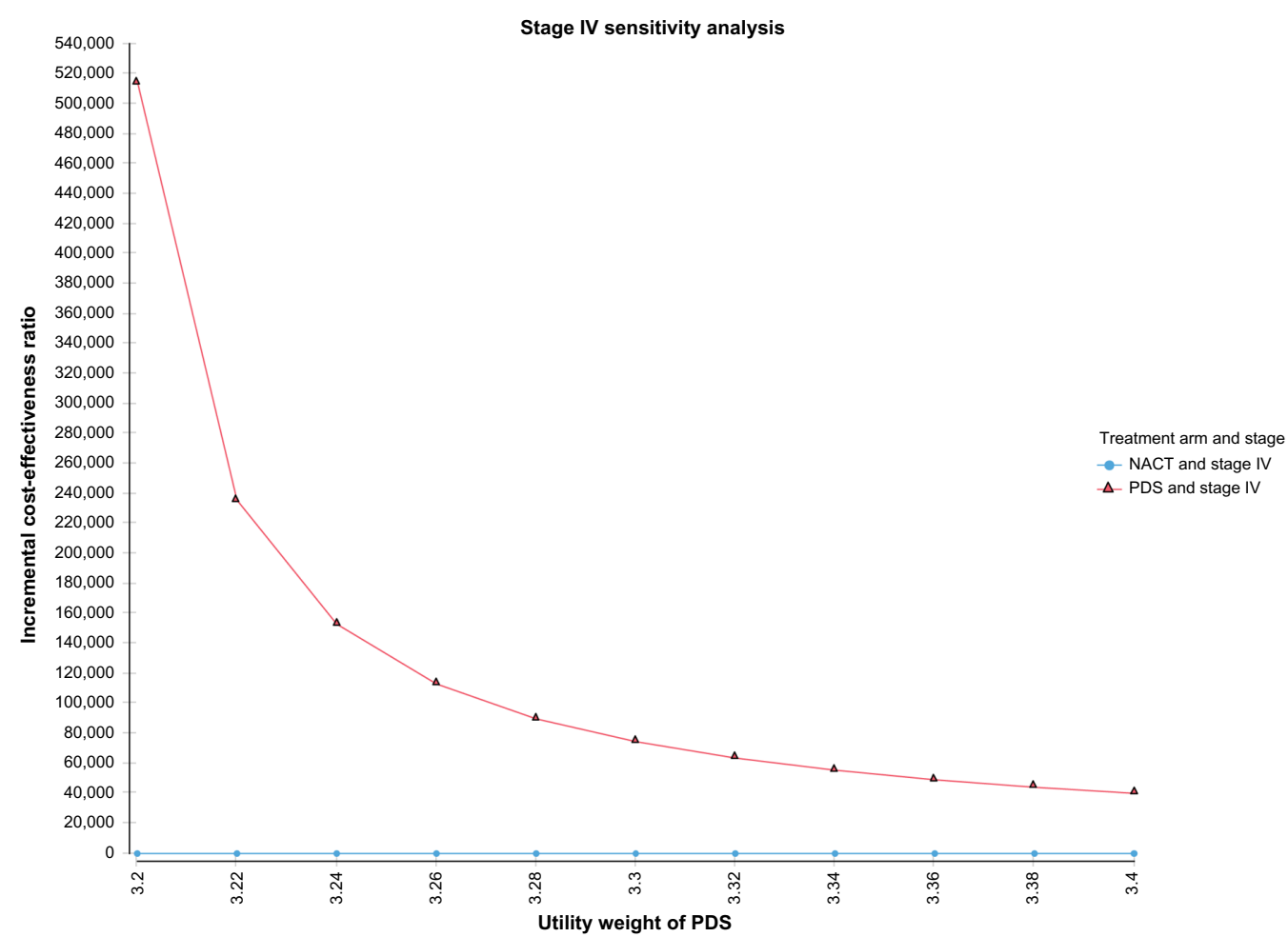

Figure 3 Univariate sensitivity analysis for the impact of the utility weight of PDS on the incremental cost-effectiveness ratio for women with stage IV ovarian cancer. Abbreviations: NACT, neoadjuvant chemotherapy; PDS, primary debulking surgery.

trial supports these conclusions and also found PDS to be associated with increased morbidity without evidence of a survival benefit. ${ }^{10}$

Conversely, Chi et al performed a retrospective study within a single institution. ${ }^{7}$ The study matched the time interval of the EORTC study with patients who underwent NACT and PDS at their institution for stage IIIC and IV ovarian cancer. The authors found an increase in overall survival in the PDS arm compared to the EORTC trial and proposed that the survival difference may be attributed to extent of disease burden, expertise of the surgeon, and patient selection to NACT or PDS. As well, this study has fallen under scrutiny for a number of reasons, too. In particular, concerns are related to the validity of retrospective analysis and that physician bias cannot be measured retrospectively. In a recent retrospective analysis of 326 women with stage IIIC and IV ovarian cancer, NACT was associated with an increased optimal debulking rate but inferior 7-year survival. Overall, they found that the 7-year overall survival for women who were optimally debulked was significantly higher in women who underwent PDS. ${ }^{25}$

Given these disparities, we felt it important to provide additional clarity to the subtle differences between PDS and NACT with respect to survival and cost. In particular, a comparison of the cost relative to effectiveness with the inclusion of quality-of-life differences was performed. In this investigation, the base case assumes a slightly improved survival for PDS over NACT in both stage IIIC and stage IV ovarian cancer with hazard ratios of 1.36 and 1.28 , respectively. NACT was slightly less costly than PDS for stage IIIC and significantly less costly for stage IV ovarian cancer. Based on this analysis, PDS is more cost-effective than NACT for the treatment of stage IIIC ovarian cancer and not as cost-effective as NACT for the treatment of stage IV ovarian cancer.

The strength of this analysis is that it includes assumptions based on a large number of women with stages IIIC and IV ovarian cancer and that the cost estimates are based on Medicare claims data linked to the SEER registry. Some of the limitations to using SEER-Medicare data are that the dataset only includes patients over 65 years, that there are more comorbidities in this population, and that generalizability to a broader population may be difficult. Additional limitations include inability to account for the extent of disease, the extent of surgery, and the amount of residual disease and inability to control for subtle unmeasured factors that may influence the decision for one treatment paradigm over the other. In addition, this study is limited by the biases that exist when using retrospective data such as selection bias and misclassification bias. Also, the same utility weight 
was used for PDS and NACT during the first year following diagnosis, which may not accurately reflect the comparative morbidity between PDS and NACT within the first year of diagnosis. A sensitivity analysis showed that, if we consider PDS to be more morbid than NACT during the first year, PDS is still more cost-effective in stage IIIC ovarian cancer. What is unclear is to what extent PDS is more morbid and what the correct utility weights for each treatment should be to accurately reflect their relative impacts on quality of life. In addition, we assumed that the cost of end-of-life care would be similar for both treatment arms and therefore did not include end-of-life costs.

\section{Conclusion}

Our analysis found that PDS for advanced ovarian cancer is more cost-effective for stage IIIC disease. In stage IV ovarian cancer, the cost/QALY exceeds a willingness-to-pay threshold of \$50,000/QALY; however, other factors used in medical decision-making should be considered as well. In the future, this may be offset by improvements in 5-year overall survival for women with advanced ovarian cancer that result from initiatives that: 1) centralize care of women with ovarian cancer to high-volume providers and medical centers; 2) improve compliance with NCCN guidelines; and 3) reduce barriers to access for disparate communities.

\section{Acknowledgments}

Dr Forde was supported by a Ruth L Kirschstein National Research Service Award Institutional Training Research Grant, number 2T32 CA06039611.

\section{Disclosure}

The authors report no conflicts of interest in this work.

\section{References}

1. Siegel R, Ma J, Zou Z, Jemal A. Cancer statistics, 2014. CA Cancer J Clin. 2014;64(1):9-29.

2. Hofstetter G, Concin N, Braicu I, et al. The time interval from surgery to start of chemotherapy significantly impacts prognosis in patients with advanced serous ovarian carcinoma - analysis of patient data in the prospective OVCAD study. Gynecol Oncol. 2013;131(1):15-20.

3. National Comprehensive Cancer Network. Clinical Practice Guidelines for Ovarian Cancer. 2012:13.

4. Hoskins WJ, McGuire WP, Brady MF, et al. The effect of diameter of largest residual disease on survival after primary cytoreductive surgery in patients with suboptimal residual epithelial ovarian carcinoma. Am J Obstet Gynecol. 1994;170(4):974-979; discussion 979-980.

5. Chi DS, Bristow RE, Armstrong DK, Karlan BY. Is the easier way ever the better way? J Clin Oncol, 2011;29(31):4073-4075.

6. Chi DS, Eisenhauer EL, Zivanovic O, et al. Improved progression-free and overall survival in advanced ovarian cancer as a result of a change in surgical paradigm. Gynecol Oncol. 2009;114(1):26-31.
7. Chi DS, Musa F, Dao F, et al. An analysis of patients with bulky advanced stage ovarian, tubal, and peritoneal carcinoma treated with primary debulking surgery (PDS) during an identical time period as the randomized EORTC-NCIC trial of PDS vs neoadjuvant chemotherapy (NACT). Gynecol Oncol. 2012;124(1):10-14.

8. Vergote I, Tropé CG, Amant F, et al; European Organization for Research and Treatment of Cancer-Gynaecological Cancer Group; NCIC Clinical Trials Group. Neoadjuvant chemotherapy or primary surgery in stage IIIC or IV ovarian cancer. $N$ Engl J Med. 2010;363(10): 943-953.

9. Kehoe S, Hook J, Nankivell M, et al. Chemotherapy or upfront surgery for newly diagnosed advanced ovarian cancer: results from the MRC CHORUS trial. Presented at: 2013 ASCO Annual Meeting; May 31-June 4, 2013 Chicago, IL, USA. Abstract 5500.

10. Wright JD, Ananth CV, Tsui J, et al. Comparative effectiveness of upfront treatment strategies in elderly women with ovarian cancer. Cancer. 2014;120(8):1246-1254.

11. Overview of the SEER Program [webpage on the Internet]. Bethesda: National Cancer Institute. Available from: http://seer.cancer.gov/about/ overview.html. Accessed January 15, 2015.

12. SEER-Medicare: Brief Description of the SEER-Medicare Database [webpage on the Internet]. Bethesda: National Cancer Institute; [updated March 2, 2015]. Available from: http://appliedresearch.cancer.gov/ seermedicare/overview/. Accessed January 15, 2015.

13. Warren JL, Klabunde CN, Schrag D, Bach PB, Riley GF. Overview of the SEER-Medicare data: content, research applications, and generalizability to the United States elderly population. Med Care. 2002;40 (8 Suppl):IV-3-18.

14. Consumer Price Index-All Urban Consumers: US Medical Care Services [webpage on the Internet]. Washington: United States Bureau of Labor Statistics. Available from: http://data.bls.gov/cgi-bin/ surveymost?cu. Accessed January 15, 2015.

15. Bristow RE, Santillan A, Salani R, et al. Intraperitoneal cisplatin and paclitaxel versus intravenous carboplatin and paclitaxel chemotherapy for stage III ovarian cancer: a cost-effectiveness analysis. Gynecol Oncol. 2007;106(3):476-481.

16. Lesnock JL, Farris C, Krivak TC, Smith KJ, Markman M. Consolidation paclitaxel is more cost-effective than bevacizumab following upfront treatment of advanced epithelial ovarian cancer. Gynecol Oncol. 2011; 122(3):473-478.

17. Messori A, Trippoli S, Becagli P, Tendi E. Treatments for newly diagnosed advanced ovarian cancer: analysis of survival data and cost-effectiveness evaluation. Anticancer Drugs. 1998;9(6): 491-502.

18. Brain KE, Smits S, Simon AE, et al; ICBP Module 2 Working Group. Ovarian cancer symptom awareness and anticipated delayed presentation in a population sample. BMC Cancer. 2014;14:171.

19. Cost-Effectiveness Analysis Registry [homepage on the Internet]. Boston: The Center for the Evaluation of Value and Risk in Health, The Institute for Clinical Research and Health Policy Studies, Tufts Medical Center. Available from: http://www.cearegistry.org. Accessed December 12, 2014.

20. Berger K, Fischer T, Szucs TD. Cost-effectiveness analysis of paclitaxel and cisplatin versus cyclophosphamide and cisplatin as first-line therapy in advanced ovarian cancer. A European perspective. Eur J Cancer. 1998;34(12):1894-1901.

21. Cohn DE, Kim KH, Resnick KE, O’Malley DM, Straughn JM Jr. At what cost does a potential survival advantage of bevacizumab make sense for the primary treatment of ovarian cancer? A cost-effectiveness analysis. J Clin Oncol. 2010;29(10):1247-1251.

22. Havrilesky LJ, Secord AA, Darcy KM, Armstrong DK, Kulasingam S; Gynecologic Oncology Group. Cost effectiveness of intraperitoneal compared with intravenous chemotherapy for women with optimally resected stage III ovarian cancer: a Gynecologic Oncology Group study. J Clin Oncol. 2008;26(25):4144-4150.

23. Havrilesky LJ, Secord AA, Kulasingam S, Myers E. Management of platinum-sensitive recurrent ovarian cancer: a cost-effectiveness analysis. Gynecol Oncol. 2007;107(2):211-218. 
24. Sfakianos GP, Havrilesky LJ. A review of cost-effectiveness studies in ovarian cancer. Cancer Control. 2011;18(1):59-64.

25. Rosen B, Laframboise S, Ferguson S, et al. The impacts of neoadjuvant chemotherapy and of debulking surgery on survival from advanced ovarian cancer. Gynecol Oncol. 2014;134(3):462-467.
26. National Cancer Institute. [homepage on the Internet]. Available from http://www.cancer.gov/. Accessed 22 February 2016. 


\section{Supplementary material}

Table SI Procedure and diagnosis codes

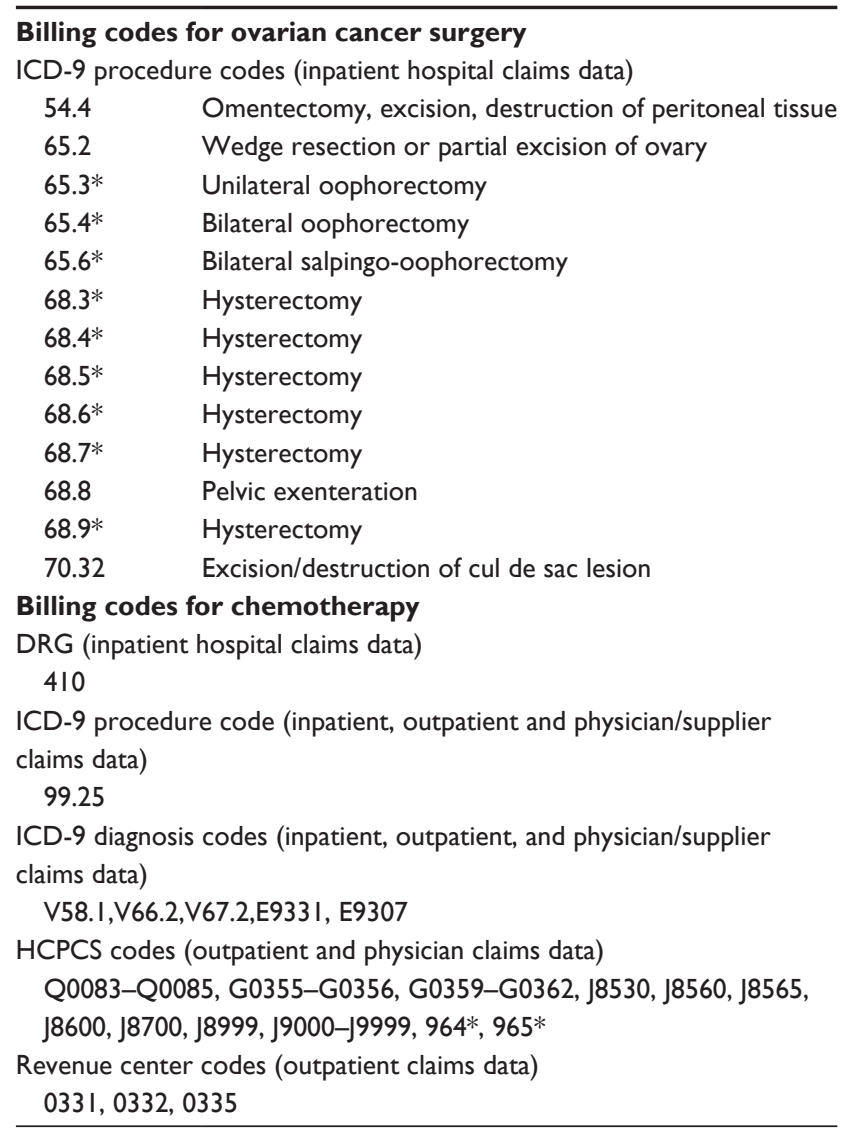

Abbreviations: DRG, diagnosis related group; HCPCS, Healthcare Common Procedure Coding System; ICD-9, International Classification of Diseases, Ninth Revision.

\section{Publish your work in this journal}

ClinicoEconomics \& Outcomes Research is an international, peerreviewed open-access journal focusing on Health Technology Assessment, Pharmacoeconomics and Outcomes Research in the areas of diagnosis, medical devices, and clinical, surgical and pharmacological intervention. The economic impact of health policy and health systems organization also constitute important areas of coverage. The manuscript management system is completely online and includes a very quick and fair peer-review system, which is all easy to use. Visit http://www.dovepress.com/testimonials.php to read real quotes from published authors. 